CULTURA, LENGUAJE Y REPRESENTACIÓN / CULTURE, LANGUAGE AND REPRESENTATION · ISSN 1697-7750 • VOL. XV \2016, PP. 11-19 REVISTA DE ESTUDIOS CULTURALES DE LA UNIVERSITAT JAUME I / CULTURAL STUDIES JOURNAL OF UNIVERSITAT JAUME I

DOI: HTTP://DX.DOI.ORG/10.6035/CLR.2016.15.1

\title{
Comunicación y cambio social. Un análisis desde la investigación centrado en el periodismo*
}

\author{
ALEX IVÁN ARÉVALO SALINAS \\ ALESSANDRA FARNÉ \\ UNIVERSITAT JAUME I DE CASTELLÓN
}

En los últimos años observamos un creciente interés por profundizar en el estudio de la comunicación y el cambio social (Gumucio-Dagron y Tufte, 2006; Marí Sáez, 2013; Nos Aldás y Santolino, 2015; Tufte, 2015). En España este crecimiento se expresa en el desarrollo de proyectos de investigación, ${ }^{1}$ la publicación de artículos, libros y capítulos, la organización de congresos y seminarios, el funcionamiento de redes de trabajo o la aplicación de este conocimiento en campañas de sensibilización, entre otros aspectos. Una muestra de esta variedad de iniciativas es la conformación del grupo de trabajo sobre comunicación y ciudadanía en la Asociación Española de Investigación en Comunicación (AE-IC); la creación de revistas científicas como Commons. Comunicación y Ciudadanía digital de la Universidad de Cádiz e Internacional de Comunicación y Desarrollo (RICD) de la Universidad de Santiago de Compostela; o la implantación de los Premios Enfoque por parte de algunas universidades españolas (Universitat Jaume I de Castellón y Universidad Carlos III) en conjunto con la Coordinadora de ONG para el Desarrollo de España. ${ }^{2}$

* Esta introducción forma parte de los resultados del proyecto de investigación «Evaluación e indicadores de sensibilidad moral en la comunicación actual de los movimientos sociales» (Ministerio de Economía y Competitividad, código CSo 2012-34066 y de los proyectos del Plan de Promoción de la Universitat Jaume I de Castellón, código P1 1B2015-21 y P1 1A 2012-05).

1. Algunos de estos proyectos son «Comunicación para el desarrollo y el cambio social en España: diseño de indicadores para la medición de su impacto social» (CsO2014-52005-R) (2015-2017) o «Evaluación e indicadores de sensibilidad moral en la comunicación actual de los movimientos sociales» (CSO2012-34066) (2013-2015), http://www.e-comunicambiosocial. uji.es/, ambas iniciativas en el marco de los proyectos de investigación del Ministerio de Economía y Competitividad de España.

2. Estos premios son una iniciativa novedosa en el contexto español, al galardonarse las buenas y malas prácticas de los medios de comunicación, sus secciones y periodistas en una votación efectuada por la ciudadanía. Más información en: http://premiosenfoque.com. 
También ha sido relevante la puesta en funcionamiento de la red \#Comunicambio en Facebook y Twitter, que se constituye en un espacio de aprendizaje mutuo donde confluyen comunicadores y activistas para compartir materiales y experiencias. Estas redes de cooperación tuvieron en 2015 un alcance presencial con la organización del congreso internacional Comunicación, Sociedad civil y Cambio social (\#Comunicambio) en la ciudad de Castellón de la Plana ${ }^{3}$ (20-22 de mayo de 2015), que congregó a más de 300 especialistas de países como España, Colombia, Dinamarca, Estados Unidos o Portugal, entre otros, y cuyas principales aportaciones se han publicado en el libro \#comunicambio (Nos Aldás, Arévalo Salinas y Farné, 2015).

En lo que respecta a la investigación, la comunicación para el cambio social presenta diferentes líneas de trabajo, las cuales se pueden agrupar a partir del tipo de medio (periodismo, publicidad o cine, entre otros). En los últimos años, esta área de estudio se ha visto influenciada por un creciente número de trabajos que abordan la influencia de las redes digitales en la transformación social, como por ejemplo en la apropiación que hace la sociedad civil de las diversas herramientas para empoderarse, fomentar sus demandas y facilitar su organización, en el marco de un contexto de movilización social en diferentes países y regiones.

En la siguiente introducción nos centraremos en el ámbito específico del periodismo y el cambio social, que engloba trabajos, prácticas e investigaciones tendientes a desarrollar unos medios periodísticos más responsables y comprometidos con la mejora de las condiciones de vida de las personas y su entorno. Estas reivindicaciones se fundamentan en un contexto de rechazo hacia algunos medios convencionales que presentan una cobertura informativa de baja calidad, al dar prioridad a temas irrelevantes como una forma de silenciar o invisibilizar los problemas estructurales. A lo anterior hay que añadir que los problemas estructurales, cuando logran ser retratados, tienen un tratamiento superficial, siendo frecuente la omisión de los factores que desencadenan la violencia y priorizándose los aspectos visibles como el número de muertos o los daños materiales. El desprestigio hacia algunos medios convencionales también se fundamenta en las relaciones de dependencia que tienen estas empresas o medios públicos con las lógicas de poder, donde se imponen controles o filtros al trabajo profesional de los periodistas, llegando incluso estas dinámicas a provocar faltas éticas. Estos aspectos quedan detallados en el documental Sombras de Libertad (Shadows of Liberty) del

3. Para más información puede consultar la página del congreso: www.comunicambio2015.uji.es. 
director Jean Philippe Tremblay y en algunas obras de referencia de autores como Chomshy y Herman (2000). ${ }^{4}$

El escenario mencionado es una muestra de que el modelo periodístico actual experimenta una crisis sistémica que abarca al menos tres dimensiones. La primera, de legitimidad y confianza por parte de las audiencias. En España, la confianza sobre la información recibida por los periodistas es de 5.3 en escala de 1 a 10, según los datos del informe anual de 2014 de la Asociación de la Prensa de Madrid (APM). La segunda dimensión se refiere a una crisis en los esquemas que se utilizan para la construcción de la noticia, que demanda nuevos parámetros (Arévalo Salinas, 2014) y, por último, una profunda crisis laboral que se observa en la precarización que experimenta este sector (bajas remuneraciones, exceso de horas de trabajo o dificultades para la formación, unido a un alto desempleo). Al respecto, la Asociación de la Prensa de Madrid (2013) señala que desde 2008 se perdieron en España 11.151 empleos y 284 medios de comunicación dejaron de funcionar.

En respuesta a este escenario, diversos sectores sociales defienden la necesidad de una reforma mediática para disponer de medios de comunicación más plurales y comprometidos, donde la responsabilidad social sea la prioridad por encima del lucro. Este ideario en España se sintetiza en el manifiesto sobre periodismo y derechos humanos firmado en 2008 por periodistas, fotoperiodistas o activistas, y a nivel internacional en el informe Mc Bride de $1980^{5}$ y sus recomendaciones para instaurar un Nuevo Orden Mundial de la Información y Comunicación (NOMIC), al amparo de la UNESCO.

La investigación teórica también se ha sumado a estas demandas desarrollando diferentes conceptos como periodismo de paz (Mc Goldrick y Lynch, 2000; Espinar y Hernández, 2012; Arévalo Salinas, 2014), periodismo preventivo (Bernabé Fraguas, 2007), periodismo público (Miralles, 2001), periodismo social (Cyntrinblum, 2007) o periodismo intercultural (Israel Garzón, 2000), entre otros. Estos conceptos se complementan con otros más amplios como comunicación para el cambio social (Figueroa, Kincaid, Rani y Lewis, 2002), comunicación para la paz (Arévalo Salinas, 2014), comunicación para el desarrollo (Cárdenas Lorenzo, 2009) o comunicación participativa (Servaes y Malikhao, 2007), entre otros. Todos estos conceptos plantean la importancia de unos discursos que permitan empoderar a las personas en su rol como ciudadanos

4. Para más información: http://www.rtve.es/television/20150204/documentos-tv-sombraslibertad/675083.shtml.

5. Se puede consultar su contenido y sus recomendaciones en el siguiente enlace: http://agmer. org.ar/index/wp-content/uploads/2014/05/Informe-MacBride-parte1.pdf. 
críticos, ya sea participando de manera activa en el debate democrático o bien facilitando la comprensión de los problemas estructurales.

Entre las diferentes líneas de investigación de un periodismo para el cambio social, o desde sus distintas denominaciones, se destaca el estudio de aquellos medios de comunicación que en sus bases o principios se autodefinen como un periodismo independiente, en relación a la influencia de los grupos de poder, y que además manifiestan un compromiso por mejorar sus sociedades. En esta línea de investigación, los trabajos pueden indagar aspectos como los esquemas utilizados para la elaboración de las noticias, sus mecanismos de organización o sus vías de financiación.

Los medios de comunicación que presentan este perfil, entre ellos el periódico digital Periodismo Humano (http://periodismohumano.com/), se caracterizan por desarrollar un enfoque de denuncia, que incluye el análisis de acontecimientos y hechos que tienen escasa cobertura o son relegados a espacios secundarios en los medios convencionales como los abusos laborales en reconocidas empresas o proyectos empresariales que infringen las leyes ambientales, entre otros. Estamos ante unos medios que revitalizan el periodismo de investigación y el análisis en profundidad.

En algunos de estos medios se aplican formas horizontales de trabajo y se establecen mecanismos de financiamiento que permiten asegurar la independencia como las donaciones, la captación de socios o una publicidad que respeta los códigos éticos. Un ejemplo en España es la revista La Marea (http:// www.lamarea.com/) que presenta un modelo de cooperativa o Periodismo Humano que es desarrollado por una asociación cultural sin ánimo de lucro.

El estudio de estos proyectos periodísticos, principalmente en aquellos que han logrado ser viables económicamente, es relevante en cuanto a las posibilidades de indagar en los criterios que explican su sostenibilidad. En España podemos definir como un ejemplo exitoso a eldiario.es, al incrementar el número de socios, de 8.703 en 2014 a 12.257 en 2015. Como consecuencia de lo anterior, este medio digital ha aumentado el número de periodistas que trabajan en su redacción de 29 en 2014 a 44 en $2015 .{ }^{6}$

En este análisis introductorio no podemos dejar de mencionar la influencia positiva que han ejercido los movimientos sociales actuales en las demandas de cambio del sistema mediático y en defender un periodismo más responsable, lo cual, incluso, se ha manifestado en el desarrollo de propuestas comunicativas gestionadas por sus propios miembros. En el caso del movimiento 15M de Espa-

6. Para más información: http://www.eldiario.es/escolar/cuentas-eldiarioes-tercer-aniversario_6_431916832.html. 
ña encontramos iniciativas periodísticas como el periódico Madrid15M (http:// madrid15m.org/), Ágora Sol Radio (http://agorasolradio.blogspot.com.es/) o el canal audiovisual Toma la Tele (http://www.tomalatele.tv/), entre otros. A los que se suman otras más amplias que favorecen la participación ciudadana en Internet como 15Mcc (http://www.15m.cc/), 15mpedia (https://15mpedia.org/ wiki/Portada) o Voces con futura (http://vocesconfutura.org/) y la transparencia del sistema democrático como Graba tu pleno (http://www.grabatupleno. com/) (Arévalo Salinas y López Ferrández, 2015).

De los proyectos comunicativos del 15M que hemos analizado (periódico Madrid15M e informativo Sí se Puede de Toma la Tele), observamos la aplicación de algunos esquemas y principios de este movimiento como la horizontalidad, donde las opiniones de todos los miembros cuentan, y el asambleísmo. En el plano discursivo, estos medios confrontan y desmitifican los marcos o relatos hegemónicos asociados a este movimiento social que tienen por objetivo desprestigiar sus demandas como una vía para desmovilizar a la ciudadanía. Un ejemplo de ello es la clásica asociación de estos movimientos sociales y sus acciones con la violencia. También en experiencias como el informativo Sí se Puede de Toma la Tele (http://www.tomalatele.tv/web/blog/category/informativos/sisepuede/) observamos cómo se potencia un estado de ánimo positivo, fomentando emociones como la esperanza, vinculada a las posibilidades de lograr modificar la situación considerada injusta. Para ello, esta propuesta periodística informa de los avances logrados por la Plataforma de Afectados por la Hipoteca ( $\mathrm{PAH}$ ), visible en el número de desahucios de viviendas suspendidos. Además, se recalca la necesidad de cooperar y participar en estas demandas. A nivel periodístico, este enfoque se plasma en la selección de sus temas, en las decisiones de edición o en el rol que desempeñan los presentadores, los periodistas activistas y sus entrevistados.

Uno de los principales aspectos del periodismo para el cambio social es la puesta en práctica de otras lógicas, esquemas y rutinas en la construcción de las noticias. Consideramos relevante que un nuevo modelo de periodismo debe posicionarse con claridad en contra de los abusos sociales que se cometen en diferentes ámbitos. De esta manera, es esencial desmitificar la regla de la objetividad que se enseña en las escuelas de periodismo como un mito de imparcialidad. El compromiso en relación a la denuncia sobre hechos y acontecimientos que vulneran los derechos humanos debe ser transversal a la línea ideológica del medio de comunicación. Considerando el amplio consenso que existe sobre la defensa de los derechos humanos en tratados, constituciones o normativas y el objetivo social que tiene la función periodística, estas empresas tienen la obligación moral de posicionarse en contra de genocidios, explotaciones laborales o situaciones 
de exclusión y discriminación, entre otras, lo que implica también realizar una amplia y profunda cobertura sobre estos hechos. Un resumen de un nuevo modelo de periodismo se detalla en Arévalo Salinas (2014), conceptualizado como periodismo de paz, el cual se resume en el siguiente cuadro:

\begin{tabular}{|c|c|}
\hline Modelo tradicional de periodismo & Modelo de periodismo de paz \\
\hline $\begin{array}{l}\text { Mito de la objetividad (argumento para } \\
\text { no mostrar un posicionamiento claro en } \\
\text { algunos temas). }\end{array}$ & $\begin{array}{l}\text { Transparencia en la subjetividad. } \\
\text { Posicionamiento claro y comprometido } \\
\text { ante las violaciones de los derechos } \\
\text { humanos. }\end{array}$ \\
\hline $\begin{array}{l}\text { Preferencia por la rentabilidad a } \\
\text { cualquier precio, incluso legitimando } \\
\text { los abusos. }\end{array}$ & $\begin{array}{l}\text { La calidad del contenido es el objetivo } \\
\text { central. La búsqueda de rentabilidad no } \\
\text { afecta las decisiones editoriales. }\end{array}$ \\
\hline $\begin{array}{l}\text { Tratamiento informativo superficial y } \\
\text { descontextualizado. }\end{array}$ & $\begin{array}{l}\text { Tratamiento informativo } \\
\text { contextualizado. Se aporta información } \\
\text { que permita comprender los } \\
\text { acontecimientos. }\end{array}$ \\
\hline $\begin{array}{l}\text { Preferencia por noticias de escasa } \\
\text { relevancia para el conjunto de las } \\
\text { sociedades. }\end{array}$ & $\begin{array}{l}\text { Inclusión de noticias de elvada } \\
\text { relevancia para las sociedades. } \\
\text { (informaciones relacionadas con } \\
\text { los problemas estructurales y la } \\
\text { transgresión de los derechos humanos). }\end{array}$ \\
\hline $\begin{array}{l}\text { Noticias negativas (catástrofes, } \\
\text { violencia, homicidios y tragedias } \\
\text { variadas). }\end{array}$ & $\begin{array}{l}\text { Equilibrio entre hechos negativos que } \\
\text { tratan los principales problemas de las } \\
\text { sociedades y los positivos (avances } \\
\text { médicos, relaciones interculturales } \\
\text { exitosas, mejoras educativas o avances } \\
\text { en acuerdos). }\end{array}$ \\
\hline $\begin{array}{l}\text { Preferencia por fuentes informativas } \\
\text { ligadas al poder y a las élites. }\end{array}$ & $\begin{array}{l}\text { Diversidad en la selección de las } \\
\text { fuentes. Inclusión de actores sociales de } \\
\text { base como oNGD, movimientos sociales } \\
\text { y sindicatos. }\end{array}$ \\
\hline $\begin{array}{l}\text { Escasa participación de la ciudadanía } \\
\text { en la selección de los temas y en las } \\
\text { decisiones de la redacción. }\end{array}$ & $\begin{array}{l}\text { Creación de mecanismos de } \\
\text { participación de la ciudadanía en las } \\
\text { decisiones editoriales y promoción } \\
\text { de dispositivos para la supervisión de } \\
\text { la ética periodística (defensores de la } \\
\text { audiencia). }\end{array}$ \\
\hline
\end{tabular}

Fuente: (Arévalo Salinas, 2014: 75) 
Sin lugar a dudas, el lograr un periodismo para la paz o de cambio social requiere de medidas estructurales, implica incentivos y sanciones, tanto a nivel regulatorio (Consejos Audiovisuales o diferentes normativas) y auto regulatorio (defensores de la audiencia, manuales de estilo o códigos éticos) como también de la propia acción ciudadana en exigir y plantear un cambio que incluya un rol vigilante de la sociedad civil (observatorios de medios de comunicación y el papel de los investigadores activistas). Por ejemplo, en el plano normativo se pueden aplicar sanciones que contrarresten las representaciones negativas, tal como acontece en el reglamento de la Ley 45 del 8 de octubre contra el racismo y toda forma de discriminación de Bolivia ${ }^{7}$ (artículo 24), que permite pagar el $50 \%$ de la sanción económica impuesta convertida en espacios donde los medios de comunicación puedan promocionar el derecho a la igualdad y la no discriminación.

Por último, destacar que el sistema educativo también debe fomentar los conocimientos y habilidades para realizar una lectura crítica de los medios de comunicación, lo que implica necesariamente conocer las estrategias discursivas de control social, y un aprendizaje sobre las pautas y criterios para plantear otros relatos y representaciones. En el caso de los comunicadores, esto tiene que ser acompañado de una interiorización de los códigos éticos de la profesión y una formación conectada con el entorno social a través de la participación en acciones comunicativas con un fuerte componente social, como la participación en radios comunitarias, en proyectos comunicativos de los movimientos sociales o campañas de las ONGD, entre otros. A pesar de su importancia, la comunicación para el cambio social, o sus diferentes concepciones, no tiene una amplia inclusión en los planes de estudio a nivel de grado y postgrado. En España, la Universitat Jaume I de Castellón cuenta con la asignatura de Comunicación para la igualdad en los grados de Publicidad y Relaciones Públicas y Comunicación Audiovisual (Gámez Fuentes, Nos Aldás y Farné, 2015) y, a nivel de máster, con las asignaturas Comunicación para la Paz y Communication for Peace (Farné y López Ferrández, en prensa) en el Máster en Estudios Internacionales de Paz, Conflictos y Desarrollo. En el contexto español, también destacamos el caso del Máster en Comunicación con fines sociales y las estrategias y campañas de la Universidad de Valladolid, campus Segovia. ${ }^{8}$

De acuerdo a estos desafíos planteados en este texto, el presente monográfico denominado «Comunicación y cambio social» pretende contribuir a

7. El reglamento se encuentra disponible en http://www.bolpress.com/art.php?Cod=2011010504.

8. Para más información sobre el estado de la cuestión de la investigación en comunicación para el cambio social y su expresión en las universidades españolas, véase Barranquero y Rosique (2014). 
ampliar las perspectivas en este campo interdisciplinar con diferentes aportaciones que incluyen revisiones conceptuales y teóricas, análisis sobre medios comunitarios y locales, una indagación sobre comunicación de los movimientos sociales o el papel de las redes sociales en los procesos de cambio social, entre otros temas.

\section{Referencias bibliográficas}

Arévalo Salinas, A. I. (2014): «Periodismo y comunicación para la paz. Indicadores y marco regulatorio», Commons: revista de comunicación y ciudadanía digital, 3 (1): 129-159.

Arévalo Salinas, A. I.; F. J. López Ferrández (2015): «Movimientos sociales y Periodismo en España. Una propuesta transversal y pacífica», Revista RIDH. Revista Interdisciplinar de Direitos Humanos, 4: 87-102.

Asociación de la Prensa de Madrid-APM (2013): Informe sobre la profesión periodística, Madrid, APM.

Asociación de la Prensa de Madrid-APM (2014): Informe sobre la profesión periodística, Madrid, APM.

Barranquero Carretero, A.; G. Rosique Castillo (2014): «La formación en comunicación/educación para el cambio social en la universidad española: rutas para un diálogo interdisciplinar», Cuadernos.info, 35: 83102.

Bernabé Fraguas, J. (ed.) (2007): Periodismo preventivo. Otra manera de informar sobre las crisis y los conflictos internacionales, Madrid, Libros de Catarata.

Cárdenas Lorenzo, L. (2009): Comunicación y construcción de ciudadanía. Aportes para el Desarrollo, Madrid, libros de Catarata.

Chomsky, N.; H. Herman (2000): Los guardianes de la libertad. Propaganda, desinformación y consenso en los medios de comunicación, Barcelona, Crítica.

Cyntrinblum, A. (2009): Periodismo social. Una nueva disciplina, Buenos Aires, La Crujía.

Espinar, E.; M. Hernández, (2012): «El periodismo de paz como paradigma de comunicación para el cambio social: características, dimensiones y obstáculos», Cuadernos de Comunicación e Información (CIC), 17: 209-237.

FArné, A.; F. J. López Ferrández (en prensa): «Comunicación para la Paz: una propuesta pedagógica para el cambio social», Opción. Revistas de Ciencias Humanas y Sociales. 
Figueroa, M. E.; L. KinCAID; M. RANI; G. LeWIS (2002): Communication for social change. Working paper serie $n^{\circ} 1$, New York, Rockefeller Foundation.

Gámez Fuentes, M. J.; E. Nos Aldás; A. Farné (2015): «Communication towards Equality in the European Higher Education Area: Building Capacities for Social Change with Spanish Undergraduates», The International Journal of Learning in Higher Education, 22 (3): 33-44.

Gumucio-Dagron, A.; T. Tufte (2006): Communication for social change anthology: Historical and contemporary readings, South Orange, NJ, Communication for Social Change Consortium.

ISRAEL Garzón, E. (2000): «Bases para el periodismo intercultural», Revista Latina de Comunicación, 34.

Marí Sáez, V. (2013): «Comunicación, desarrollo y cambio social en España: entre la institucionalización y la implosión del campo», Commons: revista de comunicación y ciudadanía digital, 2 (2): 40-64.

Nos Aldás, E.; M. SAntolino (2015): «La investigación en comunicación y cooperación en los nuevos escenarios de movilización social: ONGD, objetivos de justicia social y eficacia cultural», Revista de la Asociación Española de Investigación de la Comunicación, 2 (4): 1-7.

Nos Aldás, E.; A. Arévalo Salinas; A. Farné (2015): \#comunicambio: Comunicación y Sociedad Civil para el Cambio Social / \#com4change: Communication and Civil Society for Social Change, Madrid, Fragua.

Mc Goldrick, A.; J. Lynch (2000): «Peace journalism. How to do it», Transcend International, Recuperado de: https://www.transcend.org/tri/ downloads/McGoldrick_Lynch_Peace-Journalism.pdf. Fecha de consulta: 10-03-2015.

Miralles, A. M. (2001): Periodismo, opinión pública y agenda ciudadana, Bogotá, Grupo editorial Norma.

Servaes, J.; P. Malikhao (2007): «Comunicación participativa: ¿El nuevo paradigma?», Redes.com, 4: 43-60.

TufTe, T. (2015): Comunicación para el cambio social. La participación y el empoderamiento como base para el desarrollo mundial, Barcelona, Icaria. 